\title{
Is it Just About Physical Health? An Online Cross- Sectional Study Exploring the Psychological Distress Among University Students in Jordan in the midst of COVID-19 Pandemic
}

\author{
Ala'a B. Al-Tammemi ( $\sim$ alba0081@student.umu.se ) \\ Faculty of Medicine, Department of Epidemiology and Global Health, Umeå University, Umeå, Sweden \& \\ Doctoral School of Health Sciences, University of Debrecen, Debrecen, Hungary https://orcid.org/0000- \\ 0003-0862-0186
}

\section{Amal Akour}

Faculty of Pharmacy, Department of Pharmacy, Al-Zaytoonah University of Jordan, Amman, Jordan \& School of Pharmacy, Department of Biopharmaceutics and Clinical Pharmacy, The University of Jordan, Amman, Jordan https://orcid.org/0000-0002-2789-8514

\section{Laith Alfalah}

School of Medicine, Department of Internal Medicine, The University of Jordan, Amman, Jordan.

\section{Research Article}

Keywords: COVID-19 Pandemic, Jordan, University Students, Psychological Distress, Kessler Distress Scale.

Posted Date: October 19th, 2020

DOI: https://doi.org/10.21203/rs.3.rs-29439/v2

License: (c) (1) This work is licensed under a Creative Commons Attribution 4.0 International License. Read Full License

Version of Record: A version of this preprint was published on November 6th, 2020. See the published version at https://doi.org/10.3389/fpsyg.2020.562213. 
$1 \quad$ Is it Just About Physical Health? An Online Cross-Sectional Study

2 Exploring the Psychological Distress Among University Students in

3 Jordan in the midst of COVID-19 Pandemic

4 Ala'a B. Al-Tammemi1 ${ }^{1,2 *}$, Amal Akour ${ }^{3,4}$, Laith Alfalah ${ }^{5}$

$5 \quad{ }^{1}$ Faculty of Medicine, Department of Epidemiology and Global Health, Umeå University, Umeå,

6 Sweden.

$7 \quad{ }^{2}$ Doctoral School of Health Sciences , University of Debrecen, Debrecen, Hungary.

$8{ }^{3}$ Faculty of Pharmacy, Department of Pharmacy, Al-Zaytoonah University of Jordan, Amman, Jordan

$9{ }^{4}$ School of Pharmacy, Department of Biopharmaceutics and Clinical Pharmacy, The University of

10 Jordan, Amman, Jordan

$11{ }^{5}$ School of Medicine, Department of Internal Medicine, The University of Jordan, Amman, Jordan.

$12 *$ Correspondence:

13

Ala'a B. Al-TammemiAddress 1:Historiegränd 90734, Umeå, Sweden, Mobile: +46 73244 1350;

Email : alba0081@ student.umu.se ; Address 2:Laktanya utca 4028, Debrecen, Hungary, Mobile: +36

1620332 1362; Email : alaa.tammemi@med.unideb.hu

Keywords: COVID-19 Pandemic, Jordan, University Students, Psychological Distress, Kessler

20 Distress Scale. 
Abstract

31 Background Since the spread of COVID-19 on a global scale, most of efforts at national and

32 international levels were directed to mitigate the spread of the disease and its physical harm , paying 33 less attention to the psychological impacts of COVID-19 on global mental health especially at early 34 stages of the pandemic.

Objectives This study aimed to assess and explore (i) The levels of psychological distress and its correlates (ii) Motivation for distance learning (iii) Coping activities and pandemic related concerns, among university students in Jordan in the midst of COVID-19 pandemic

Methods A cross-sectional study was conducted using an online self-administered questionnaire. The measure of psychological distress was obtained using the 10-item Kessler Psychological Distress Scale, while other questions have explored our study's second and third aims.

Results A total of 381 completed questionnaires were included in the analysis. Female participants slightly predominated the sample $(\mathrm{n}=199,52.2 \%)$. The respondents aged $18-38$ years (mean 22.6 years, SD: 3.16). Concerning distress severity, most of respondents were regarded as having severe psychological distress $(n=265,69.5 \%) .209$ students $(54.9 \%)$ reported that they had no motivation for distance learning. Ordinal logistic regression revealed a significant correlation between distress severity and many predictors. Among the predictors that were found to act as a protective factors against higher levels of distress included older age (aOR=0.64, $\mathrm{P}=0.022$; 95\% CI: 0.44 - 0.94) , and having a strong motivation for distance learning ( $\mathrm{aOR}=0.10, \mathrm{P}=0.048 ; 95 \% \mathrm{CI}$ : 0.01 - 0.96).In contrary, being a current smoker $(\mathrm{aOR}=1.99, \mathrm{P}=0.049$; 95\% CI: 1.10 - 3.39), and having no motivation for distance learning $(\mathrm{aOR}=2.49, \mathrm{P}=0.007 ; 95 \% \mathrm{CI}$ : 1.29 - 4.80) acted as risk factors for having higher levels of psychological distress among the students .The most common coping activity reported was spending more time on social media platforms $(n=269,70.6 \%)$, and 209 students $(54.9 \%)$ reported distance learning was their most distressing concern.

Conclusion The COVID-19 pandemic and related control measures could impact the mental health of individuals, including students. We recommend a nationwide psychological support program to be incorporated into Jordan's preparedness plan and response strategy in combating the COVID-19 pandemic. 
60 COVID-19 is a highly transmissible respiratory disease caused by a new type of human coronaviruses;

61 SARS-CoV-2 (Al-Tammemi, 2020). Since its discovery in late December 2019, the disease has spread 62 widely across many countries and territories on a global scale. As of September 20,2020 more than 30 63 million confirmed cases, and over nine hundred thousand confirmed deaths across 216 countries and 64 territories were attributed to the COVID-19 (World Health Organization, 2020).

65 Epidemics and outbreaks can pose profound impacts on physical health, mental health as well as the 66 global economy resulting in disruptions of humans' daily life (Chakraborty and Maity, 2020). The containment measures that were adopted by many countries worldwide in combating the COVID-19 such as quarantine, countries' lockdown, travel restrictions, physical distancing, social isolation as well as local restrictions on individuals' mobility, can lead to a significant burden on mental health causing emotional and behavioral changes (SAMHSA, 2014; Brooks et al., 2020; Cao et al., 2020; Center for the Study of Traumatic Stress, 2020; Holmes et al., 2020).

In addition, the psychological impacts of outbreaks are considered a threat not only on individuals with pre-existing psychiatric illness but also on those who are free of any psychiatric condition (Ho et al., 2020). The fear of an epidemic can afflict individuals irrespective of their gender, age, race, or socioeconomic status. Anxiety, insomnia, anger, loneliness, fear, shame, helplessness, blame, guilt, and stigma were all found to be present during infectious diseases' outbreaks (Ho et al., 2020; Ornell et al., 2020). Different psychiatric conditions, including depression, panic attacks, Post Traumatic Stress Disorder, and even suicidality, were also reported to be associated with outbreaks, especially in younger age groups (Ho et al., 2020).

In epidemics, certain groups in the society such as older people, children, health care workers, infected patients, patients with pre-existing psychiatric conditions and students are at a greater risk of suffering from a significant degree of psychological pressure and stress compared to other individuals (Ho et al., 2020). It is essential to gather information about the impacts of the COVID-19 pandemic on the mental

84 health of the general population and specific vulnerable groups, and this will help in developing appropriate interventions that would mitigate such pandemic's adverse effects (Holmes et al., 2020). Since the beginning of the COVID-19 pandemic, most of the global efforts act on the biological and physical aspects of the pandemic in order to limit its spread within the communities. However, much 
less attention was paid to the mental health risks of the COVID-19 pandemic especially at early stages

89 of the pandemic.

90 Jordan is amongst the countries that have been struck by the COVID-19 pandemic, and in response to 91 that, many preventive and control strategies were enforced by the government to retard the viral spread 92 in the country. One of Jordan's public health responses during early stages of the pandemic was

93 declaring the closure of all schools and higher academic institutions with shifting to online remote 94 learning since the middle of March 2020 (Al-Tammemi, 2020; Jordanian Ministry of Health, 2020; 95 Prime Ministry of Jordan, 2020). The COVID-19 pandemic alongwith the disruptions that happened 96 in various sectors including the academic sector has forced the students to live in a new experience at both academic and personal levels. Consequently and in light of limited literatures that assessed mental health status of university students in Jordan, our present study aimed at (i) Exploring the level of

99 psychological distress and its correlates amongst university students during the COVID-19 pandemic

100 (ii) Evaluating the students' motivation for distance learning and, (iii) Exploring coping activities and major pandemic related concerns from students' perspective.

\section{MATERIALS AND METHODS}

\section{$103 \quad 2.1 \quad$ Study Design and Participants}

104 A cross-sectional study was conducted in May 2020, using an online self-administered questionnaire 105 of closed-ended questions. The participants in our study were recruited through social media platforms 106 employing a convenience sampling strategy. The questionnaire was distributed across seven randomly 107 selected Facebook groups of university students in Jordan and academic groups on WhatsApp 108 messenger for a duration of one day. These social media groups were created by students as a tool for 109 general and academic communication within the students' community and involved students who are 110 currently enrolled in different study programs and levels at various academic institutions in Jordan.

111 The students who were available and voluntarily willing to be involved in the study could open a link

112 to get an information letter about the study, eligibility criteria, and informed consent as a prerequisite 113 to proceed in participation.Considering the nature of the web-based Google form surveys, the students 114 were instructed to fill out the questionnaire with probity after fullfilling the eligibility criteria, 115 consenting on voluantry participation and filling it only once. We did not provide any form of 116 compensation to the participants upon their involevement in our study. 
117 We decided to carry out this study using an internet-based survey due to the current pandemic crisis

118 and the national strict measures on the face to face communication coupled with the closure of all

119 academic institutions in Jordan at the time of data collection In addition, using the internet and social

120 media for the recruitment and sampling procedures in this study has shown to be an effective and time-

121 efficient method to reach inaccessible potential participants from different Jordanian regions by

122 eliminating any geographical boundaries. A recent systematic review of 109 published articles that

123 aimed at evaluating the use of social media such as Facebook for recruitment of research participants

124 in various psychological and medical studies came into evidence, which supported the effectiveness

125 and efficiency of this strategy (Thornton et al., 2016).

126 For a student to be able to participate in this study, all the following eligibility criteria were 127 implemented:

128 1- Age $\geq 18$ years

129 2- Residing in Jordan during the pandemic crisis

130 3- Active enrollment in an undergraduate or postgraduate study at a Jordanian University.

\section{$132 \quad 2.2 \quad$ Instruments and Measures}

133 The online questionnaire was created using Google Forms provided by Google TM and was constructed

134 in modern standard Arabic. The questionnaire consisted of three sections, with a total of 24 questions.

135 The first section comprised of seven questions about sociodemographic information including age,

136 gender, region of residence, study level, type of academic institution, marital status, and smoking status

137 alongwith two questions about any history of pre-existing psychiatric conditions and related 138 medication use.

139 The second section included an Arabic version of the 10-item Kessler Psychological Distress Scale 140 (K10). This Arabic version was translated from the original English version by a team of linguistic 141 experts from multiple Arab countries (Egypt, Libya, Lebanon, and Tunisia) in addition to Arab experts 142 in Psychology in the United States. The Arabic version is provided by Harvard Medical School on the 143 webpage of the National Comorbidity Survey (National Comorbidity Survey, 2013).

144 The 10-item Kessler Psychological Distress Scale (K10) is an internationally validated tool for simple 145 and rapid assessment/screening of non-specific psychological distress in which 10 questions with 5- 
146 point Likert scale responses are present (Andrews and Slade, 2001; Kessler et al., 2002; Fassaert et al., 147 2009; Easton et al., 2017). On a sample of Arabs, the Arabic version of the 10-item Kessler 148 Psychological Distress Scale (K10) has shown satisfactory psychometric properties with high internal 149 consistency and reliability (Cronbach's $\alpha=0.88$ ) (Easton et al., 2017).

150 The questions of the 10-item Kessler Psychological Distress Scale (K10) are:

151 Question 1 (Q1). "During the last 30 days, about how often did you feel tired out for no good reason?"

152 Question 2 (Q2). "During the last 30 days, about how often did you feel nervous?"

153 Question 3 (Q3). "During the last 30 days, about how often did you feel so nervous that nothing could 154 calm you down?”

155 Question 4 (Q4). "During the last 30 days, about how often did you feel hopeless?"

156 Question 5 (Q5). "During the last 30 days, about how often did you feel restless or fidgety?"

157 Question 6 (Q6). "During the last 30 days, about how often did you feel so restless you could not sit 158 still?"

159 Question 7 (Q7). "During the last 30 days, about how often did you feel depressed?"

160 Question 8 (Q8). “During the last 30 days, about how often did you feel that everything was an effort?"

161 Question 9 (Q9). "During the last 30 days, about how often did you feel so sad that nothing could 162 cheer you up?"

163 Question 10 (Q10). "During the last 30 days, about how often did you feel worthless?"

164 The response choices with their correspondence score weights are None of the time (1 point), A little 165 of the time (2 points), Some of the time (3 points), Most of the time (4 points), and All the of time (5 166 points). With having 10 questions and five weighted responses as previously described, the total 167 minimum and maximum scores for the Kessler distress scale (K10) are 10 and 50, respectively. As per 168 the scale's guide, $\mathbf{Q 3}$ and $\mathbf{Q 6}$ were not asked in our study and were automatically scored as one point 169 if the preceding questions Q2 and Q5 were answered as None of the time. 
170 The severity of psychological distress was then categorized into four groups as the following based on

171 the total K10 distress score for each participant: 10-19 = no psychological distress, 20-24 = mild 172 psychological distress, 25-29 = moderate psychological distress, and 30-50 = severe psychological 173 distress (Andrews and Slade, 2001).

174 The third section of the questionnaire included five questions about the following topics: one question 175 about coping activities during COVID-19 pandemic and the nationwide curfew in Jordan. This question 176 included a list of 13 activities from which the students were able to choose all that applies to their 177 situation and to add any activity that was not listed among the choices using the option "others, please 178 specifiy”. Most of the listed acitivities were suggested by the authors and few others were adapted from another resource (USCF, 2020).Amongst these activities were spending more time on social networking platforms ,talking to friends, watching television, more engagement with family, listening to music, practicing sports at home, studying and preparing for exams, increase smoking, reading 182 Books / novels, meditation, herbal drinks, practicing Yoga, talking to a psychological counselor and others. Two questions about the use of medications to cope with COVID-19 related distress, in which one of the questions was with yes/no response to know whether the student used a medicinal drug to cope with pandemic distress or not and if yes, to report the frequency of usage, while the other question included different classes of medications with examples on most common trade names in each class and the students could add any medication that was not listed using the option "others, please specifiy". Additionally, one question about students' motivation for online distance learning, using a single-answer item with responses as no motivation, low motivation, moderate motivation, and strong motivation, and lastly, a question about major pandemic related concerns as perceived by the students.

191 This question was a single-answer question with five response choices including being infected by COVID-19, online distance learning, the economic impacts of the COVID-19 pandemic, curfew and social isolation, and other concerns.

The questionnaire was piloted on 10 students who were approached by the first author to test the phrasing, suitability, and understandability of the questions. The responses from these 10 students, as well as incomplete questionnaires, were excluded from the analysis. 


\subsection{Data Management and Analysis}

201 Completed questionnaires were extracted from Google Forms as an Excel sheet and were then

202 incorporated into STATA IC 16.1 (StataCorp LLC., Texas, USA). Descriptive analysis and summary

203 statistics were used in which numerical variables were described as mean and standard deviation, while 204 categorical variables were described as frequency and percentage. In addition, non-parametric tests 205 were used including Wilcoxon Rank-Sum test to compare the mean of total K10 distress scores 206 between males and females while Spearman's rank correlation to test the relationship between age and 207 total K10 distress scores. Besides, ordinal logistic regression was employed to assess the correlation 208 between psychological distress severity (outcome variable with ordinal responses) and other 209 independent sociodemographic predictors. The confidence level was set at $95 \%$ and a $P$-value less than $210 \quad 0.05$ was considered statistically significant.

\section{$211 \quad 2.4 \quad$ Ethical considerations}

212 The study was conducted according to the Declaration of Helsinki. Ethical approval was granted by 213 the Institutional Review Board at Al-Zaytoonah University of Jordan. Besides, the questionnaire 214 ensured the privacy and confidentiality of participants by not asking any questions about names, phone 215 numbers, physical addresses, or emails; thus, all participants were anonymous. Also, an information 216 letter was incorporated into the first page of the questionnaire and included explicit information about 217 the researchers and their affiliations, the study description and objectives, eligibility criteria for 218 participation, voluntary participation and withdrawal, benefits and risks, privacy and confidentiality 219 aspects, data handling, as well as the contact details for any enquiry. Furthermore, at the end of the 220 information letter, electronic informed consent was requested from participants as a prerequisite to join 221 the survey voluntarily. 


\subsection{Respondents' Characteristics}

230 A total of 397 questionnaires were received, and 16 were excluded due to incompleteness. So, the

231 remaining 381 were included in our analysis. There was a slight predomination of female participants $232(\mathrm{n}=199,52.2 \%)$ compared to male participants $(\mathrm{n}=182,47.8 \%)$. The mean age was 22.6 years $233(\mathrm{SD}=3.16)$ and ranged between $18-38$ years. The vast majority of participants were single $(\mathrm{n}=352$, $23492.4 \%)$, undergraduates $(n=323,84.8 \%)$, studying at governmental/public universities or colleges $235(\mathrm{n}=209,54.9 \%)$, living in the central region of Jordan $(\mathrm{n}=302,79.3 \%)$, currently non-smokers $(\mathrm{n}=267$ $236,70.1 \%)$ as well as with no history of pre-existing psychological or mental illness $(\mathrm{n}=366,96.1 \%)$. 237 More details about the sociodemographic characteristics of the respondents are provided in Table 1.

\subsection{Kessler Psychological Distress Scale (K10) Results}

240 The total K10 distress scores had a mean of 34.2 (SD=9.4). The mean K10 distress score was slightly 241 higher among women (mean=34.7, $\mathrm{SD}=8.56$ ) compared to men (mean=33.7, $\mathrm{SD}=10.3$ ); however, 242 Wilcoxon Rank-Sum test showed that this difference is statistically insignificant $(P=0.566)$. 243 Concerning age, Spearman's rank correlation test revealed a statistically significant inverse 244 relationship between age and total $\mathrm{K} 10$ distress score $(\mathrm{Rho}=-0.1645, P=0.001)$, which indicates that 245 younger age groups were more likely to have higher total K10 distress scores; thus, more distress. 246 (Figure 1).

Regarding psychological distress severity categorization and based on K10 scale's categories described earlier in study instrument, most of respondents were regarded as having severe psychological distress $(\mathrm{n}=265,69.5 \%)$, followed by moderate psychological distress $(\mathrm{n}=48,12.6 \%)$, mild psychological distress ( $\mathrm{n}=41,10.8 \%)$, and no psychological distress $(\mathrm{n}=27,7.1 \%)$. Tables 2 and Figure 2 show more descriptive results of the K10 distress scale by severity level and gender.

Ordinal logistic regression was employed to assess the correlation between distress severity (ordinal outcome variable) and other sociodemographic predictors, however, considering our sample size $(\mathrm{n}=381)$ and in order to achieve sufficient statistical power for the regression test, we have merged no distress and mild distress in one ordinal category as well as moderate distress and severe distress together in another ordinal category. Therefore, we had an ordinal outcome variable with two severity 
levels/categories. After that, we tested each independent variable against the outcome variable .All independent variables that had a $\mathrm{P}$ value less than 0.25 in univariable regression were included in the final ordinal logistic regression model. The regression model revealed a significant correlation between distress severity and some predictors. Among the predictors that were found to act as a protective factor against higher levels of distress were older age (aOR=0.64, $\mathrm{P}=0.022$; 95\% CI: 0.44 0.94) , and having a strong motivation for distance learning ( $\mathrm{aOR}=0.10, \mathrm{P}=0.048$; $95 \% \mathrm{CI}$ : 0.01 0.96) .In contrary, being a current smoker ( $\mathrm{aOR}=1.99, \mathrm{P}=0.049 ; 95 \% \mathrm{CI}: 1.10$ - 3.39), and having no motivation for distance learning $(\mathrm{aOR}=2.49, \mathrm{P}=0.007 ; 95 \% \mathrm{CI}: 1.29$ - 4.80) acted as risk factors for having higher levels of psychological distress among the students The detailed results of oridinal logistic regression are presented in Table 3

\subsection{The motivation for Distance Learning}

269 Surprisingly, a significant proportion of the students have reported that they had no motivation at all toward the online distance learning $(\mathrm{n}=209,54.9 \%)$, and as described earlier, students with no motivation for distance learning were more likely to suffer from higher degrees of psychological distress $(\mathrm{aOR}=2.49, \mathrm{P}=0.007$; 95\% CI: 1.29 - 4.80). Table 4 and Figure 3 demonstrate more descriptive details about the motivation for distance learning .

\subsection{Coping Activities and Concerns during the COVID-19 Pandemic}

The students have selected many coping activities that the they frequently practiced during the nationwide curfew in Jordan. Interestingly, the responses with highest frequencies were spending more time on social networking platforms like Facebook and Instagram (n=269, 70.6\%), talking to friends on mobile phones and internet $(\mathrm{n}=217,57 \%)$, watching television and movies $(\mathrm{n}=210,55.1 \%)$, more engagement with family $(\mathrm{n}=202,53 \%)$, and listening to music $(\mathrm{n}=162,42.5 \%)$. More details about these activities are provided in Table 5.

282 In addition, among the 381 respondents, 332 students (87.1\%) reported no use of any medications during the last 30 days for coping with the distress accompanied the COVID-19 pandemic and the nationwide curfew, while 49 students $(12.9 \%)$ reported the use of various types of medications at different frequencies with occasionally (1-2 times in a month) as the most common frequency. Sedative 
hypnotics (38\%) reported being on the top of the used medications followed by others (28\%), which included over-the-counter medications like Paracetamol and other simple analgesics. More details are demonstrated in Figures 4, 5, and Table 6.

Moreover, 209 students (54.9\%) reported that online distance learning was the highest and most serious issue of concern, followed by 75 students (19.7\%) who reported curfew and social isolation as their highest issue of concern. Unexpectedly, only 53 students (13.9\%) reported being infected by COVID-19 as their most serious concern. Figure 6 for more illustration.

\section{DISCUSSION}

In our study, the vast majority of the students (92.9\%) suffered from different levels of psychological distress ranging from mild to severe degrees during the COVID-19 pandemic.The psychological wellbeing of university students in the midst of the current pandemic has been established and reported in recently published litratures as well .A recent study which was conducted by Cao et al. (2020) in China and aimed at exploring the psychological impact of COVID-19 on college students using the 7item Generalized Anxiety Disorder Scale (GAD-7) has revealed that 24.9\% of students suffered from anxiety during this pandemic with a positive association of the level of anxiety with different economic and academic stressors (Cao et al., 2020). Similar to Cao et al. (2020) study, our study found that there was no significant difference in the total psychological distress scores between men and women. In addition, Cao et al. found that social support was negatively associated with anxiety status among students, and we have that as one of our most reported coping mechanisms, i.e., socialization through social networking sites. Nevertheless, in our study, age was statistically and significantly associated with distress severity; i.e the younger the age, the more likely to suffer from higher levels of psychological distress. The difference in distress proportions between our study (92.9\%) and Cao et al study (24.9\%) could be attributed to the use of different scales, i.e. GAD-7 vs. K10 as well as the sample size. In addition, we carried out the survey in a period close to final examinations, which might

311 have had an additional negative impact on the students' psychological status.

312 Additionally, a recent study by Olimat et al (2020) (Olaimat et al., 2020) was conducted to assess 313 attitudes, anxiety, as well as behavioral practices among university students in Jordan amidst the

314 COVID-19 pandemic using an online survey developed by the authors to serve their study objectives .

315 The study has found that $69.2 \%$ of participants reported being anxious as a result of fear of infection 
by COVID-19 and resultant disruptions in their lives.Among the predicitve factors that affected the

317 students' anxiety levels were age, gender and academic discipline of their study programs.Older

318 students and female students were found to have more anxiety due to the fear of infection. However,

319 in our present study the mean total K10 distress score was higher among women compared to men ,but

320 this difference in means was statistically not significant. In contrary, older age was amongst the

321 protective factors against higher levels of psychological distress in our present study.

322 Moreover, a cross sectional study was conducted in Turkey which aimed at assessing anxiety status

323 of university students using an online survey. The measure of anxiety levels were obtained using the

324 Turkish version of abbreviated Beck Anxiety Inventory.The study has found that $44 \%$ of students

325 reported a moderate level of worrisome and fear of catching COVID-19, while $80 \%$ of students

326 reported a 'severe level' of scare and worries about thier close relatives' health .The authors expected

327 that the high levels of anxiety among the students in their study could be attributed to shifting to online

328 learning alongwith other pandemic control measures such as social isolation and financial constraints

329 (Akdeniz et al., 2020). Similarly, the aforementioned worries were also reported by the students in our

330 study as part of their major concerns during the COVID-19 pandemic.

331 Furthermore, Stress and anxiety were assessed in France among university students during the current 332 pandemic.University students were asked to complete the World Mental Health International College 333 Student survey which was distributed as an online survey.Among the 291 participants in the study, the 334 majority of them experienced significant proportions of psychological distress of which $60.2 \%$ of 335 students reported escalation of their anxiety to moderate-severe levels during the COVID-19 336 pandemic.However, $82.2 \%$ of the students in our study reported moderate to severe distress. This 337 difference in distress protportions coud be as a result of different scales used and cross-cultural 338 factors.In the same study, the researchers found significant factors that affected the students' anxiety 339 level including the economic situation of the students, and the disruptions in students' life (Husky et 340 al., 2020). These factors were also reported in our study as pandemic induced concerns as perceived 341 by the students. However, in our study, we have not collected data about the students' or their families

342 financial status ; thus we could not consider it in our regression analysis to examine its influence on 343 the distress levels.

344 Jordanian universities have been taking humble attempts to implement distance learning into their 345 educational system since 2015 .Nevertheless, this strategy has been considered as a "challenging 346 pedagogy" of the learning system in the country due to many obstacles (Al-Jaghoub et al., 2009; Atoum 
et al., 2017; Al Nawas, 2020). During the COVID-19 pandemic and after realising the need to

348 implement an emergency distance learning strategy, more serious steps were taken by decision makers

349 at higher education sector and the Jordanian universities trying to guarantee a smooth shifting process

350 coupled with ensuring a quality education as well. Besides, psychological distress was reported to be

351 associated with distance learning and working from homes during the current pandemic. A recent

352 qualitative study has addressed many of the distance learning's challenges including personal,

353 technological, course-related as well as cultural challenges (Almaiah et al., 2020). These challenges

354 might explain why most of the students $(n=209,45.9 \%)$ in our study resported the lack of motivation

355 for distance learning, especially within the Jordanian context where most of educational activities were

356 used to be delivered by in-person attendance to universities/colleges with less attention to distance

357 learning.

358 Besides, smoking exhibited a risk factor for suffering from higher levels of psychological distress 359 among the students in our study, and this could be explained by the bi-directional relatiohship between 360 smoking and mental wellbeing as addressed previously in a longitudinal study in Australia (Leung et 361 al., 2012). Emotional and behavioural reactions toward the COVID-19 pandemic could vary. The type 362 of coping strategy and the extent of adopting it also differs between individuals . In the present study, 363 some students (12.9\%) reported the use of various medicinal drugs as a result of pandemic induced 364 distress. Although the figure is small but this raises a concern about the psychosocial response of some 365 individuals in response to crisis which might lead to a risky behaviour such as substance abuse 366 .Therefore, more serious efforts should be done to spread awareness about healthy coping styles among 367 different social components of the community (Pfefferbaum and North, 2020).

368 To the best of our knowledge, this is the first study in Jordan to assess the psychological distress 369 among university students using the 10-item Kessler Psychological Distress Scale (K10) during the COVID-19 pandemic. In addition, this study is amongst the limited litratures to highlight the distressing concerns brought about by online distance learning on university students in Jordan. Still, there are limitations that should be carefully taken into consideration when interpreting the results and include (i) using a non-probabilistic convenience sampling, which affects the representativness of our sample and limits the generalizability of our results. However, this sampling strategy was believed to fit in lieu of the current circumstances of the nationwide curfew, the closure of all universities and colleges in the country and shifting to online platforms (ii) the majority of respondents were undergraduates; we could have seen different results if our sample had more postgraduate students, 
and (iii) We had a relatively small sample size which could be attributed to the limited period of data collection .There was a technical difficulty to follow up the survey and keep it visible to students within social media groups due to the large number of academic enquiries posted on these groups ;thus, enforced our survey link to lose its visibility among the numerous recent posts .Also, the busy schedule of students (in the midst of a new distance learning strategy) might have affected their interests to participate in the survey (iv) The survey represented self reported states thus, over reporting or underreporting of psychological status could be expected, (v) The inherent limitation of crosssectional studies which prevent assessing temporality of events i.e psychogoligcal distress could be present prior to the pandemic and just escalated during it, and lastly, (vi) We missed the perspectives of non-arabic speaking students in Jordan as the questionnaire was designed in Arabic only. Nevertheless, findings from our study shed the lights on various degrees of psychological distress that the university students have experienced during the current pandemic, and they could be considered as a vulnerable group. Also, the findings of our study encourage for further follow up research on this topic using a nationally representative sample of university students with more specific scales for psychological distress symptoms.

393 The results of this study provide new insights to direct policy makers and decision makers in the fields of higher education, as well as mental health. More attention and monitoring of college students' mental health should be sought. Since distance learning was the highest reported concern among students, faculty members should implement effective methods to make distance learning more interactive and students friendly. Psychological interventions should be implemented by psychologists and psychiatrists to provide guidance, psychoeducation, and mental health counseling to university students. There should be more active involvement with students' psychological health, coupled with educating them on how to deal with psychological distress during unprecedented situations like the current pandemic.

At the current circumstances of COVID-19 preventive measures in Jordan (curfew and social distancing), psychological support could be provided to university students through publicly available online videos, television programs, and online/phone consultations. Also, mental health support could be provided through a hotline service to provide students with instructions about dealing with their academic stressors and other related mental health issues during this pandemic. on how to access only evidence-based information from reliable resources about the pandemic. 
Besides, a comprehensive nationwide psychological support program should be developed and

410 incorporated into Jordan's response strategy in combating the COVID-19. Future studies should assess

411 the effect of implementing these suggested interventions on students' mental health. Furthermore, as

412 the levels of psychological distress are expected to be dynamic over the upcoming period, it is wise to

413 monitor and assess the impact of easing up the governmental restrictions, i.e. ending the curfew and

414 returning to on-campus teaching, on the levels of psychological distress and anxiety among university

415 students in Jordan.

\section{CONCLUSION}

417 The control and preventive measures that are implemented during the COVID-19 pandemic resulted 418 in a severe disruption of various human life activities. The fear of the infection itself, along with the 419 strict public health measures could impact the mental health of individuals. Our study highlighted a 420 significant psychological distress among university students in Jordan during the COVID-19 pandemic and its related control measures.. A significant proportion of the students were highly concerned about and distressed by the distance learning strategy; thus, prompt actions should be taken to improve the distance learning experience and solve any associated technostress. In addition, a nationwide psychological support program should be incorporated into Jordan's preparedness plan and response strategy in combating the COVID-19 pandemic and other crisis, considering students and other vulnerable groups in the community.

\section{Conflict of Interests}

429 The authors have no conflicts of interest to declare that are relevant to the content of this article.

\section{$430 \quad$ Funding}

431 No funding was received to assist with the preparation of this manuscript. Publication charges for this article were waived by the Frontiers in Psychology Journal due to the ongoing pandemic of COVID-

\section{Acknowledgments}

435 The authors provide their sincere appreciation to university students who participated in the online survey. Also, Al-Tammemi gratefully acknowledges the funding received from The Swedish Institute toward his study at the Department of Epidemiology and Global Health, Umeå University as well as the funding received from Tempus Public Foundation toward his doctoral study at the Doctoral School of Health Sciences, University of Debrecen. The funders had no role in the study design, data collection and analysis, decision to publish, or preparation of the manuscript. 
Al-Tammemi conceptualized the study, designed, and prepared the questionnaire with inputs from Akour and Alfalah. Data collection was carried out by Al-Tammemi and Akour. The statistical analyses were done by Al-Tammemi.

Alfalah wrote the introduction. Al-Tammemi wrote the methods, materials, and results. Al-Tammemi and Akour wrote the discussion. All authors have substantially and critically contributed to editing and revising the manuscript and providing critical feedback. All authors have approved the submission of this version of the manuscript.

\section{References}

Andrews, G., and Slade, T. (2001). Interpreting scores on the Kessler Psychological Distress Scale

Akdeniz, G., Kavakci, M., Gozugok, M., Yalcinkaya, S., Kucukay, A., and Sahutogullari, B. (2020). A Survey of Attitudes, Anxiety Status, and Protective Behaviors of the University Students During the COVID-19 Outbreak in Turkey. Front. Psychiatry 11, 695. doi:10.3389/fpsyt.2020.00695.

Al-Jaghoub, S., Al-Yaseen, H., Hourani, M., and El-Haddadeh, R. (2009). E-learning adoption in higher education in Jordan: vision, reality and change. in European and Mediterranean Conference on Information Systems - EMCIS 2009 Available at: https://bura.brunel.ac.uk/bitstream/2438/4044/1/plugin-C83.pdf.

Al-Tammemi, A. B. (2020). The Battle Against COVID-19 in Jordan : An Early Overview of the Jordanian Experience. Front. Public Heal. 8, 188. doi:10.3389/fpubh.2020.00188.

Al Nawas, B. (2020). Higher Education Council reviews distance learning experience. The Jordan Times. Available at: http://jordantimes.com/news/local/higher-education-council-reviewsdistance-learning-experience.

Almaiah, M. A., Al-Khasawneh, A., and Althunibat, A. (2020). Exploring the critical challenges and factors influencing the E-learning system usage during COVID-19 pandemic. Educ. Inf. Technol. doi:10.1007/s10639-020-10219-y.

476 Cao, W., Fang, Z., Hou, G., Han, M., Xu, X., Dong, J., et al. (2020). The psychological impact of the 
COVID-19 epidemic on college students in China. Psychiatry Res. 287.

Center for the Study of Traumatic Stress (2020). Psychological Effects of Quarantine During the https://www.cstsonline.org/assets/media/documents/CSTS_FS_Psychological_Effects_Quaranti ne_During_Coronavirus_Outbreak_Providers.pdf [Accessed May 5, 2020].

Chakraborty, I., and Maity, P. (2020). COVID-19 outbreak: Migration, effects on society, global environment and prevention. Sci. Total Environ. 728, 138882. doi:10.1016/j.scitotenv.2020.138882.

Easton, S. D., Safadi, N. S., Wang, Y., and Hasson, R. G. (2017). The Kessler psychological distress scale: Translation and validation of an Arabic version. Health Qual. Life Outcomes 15, 215. doi:10.1186/s12955-017-0783-9.

Fassaert, T. J. L., Wit, M. A. S. De, Tuinebreijer, W. C., Wouters, H., Verhoeff, A. P., Beekman, A. T. F., et al. (2009). Psychometric Properties of an interviewer- administered version of the Kessler Psychological distress scale (K10) among dutch, moroccan and turkish respondents. Int. J. Methods Psychiatr. Res. 18, 159-168. doi:10.1002/mpr.288.

Ho, C. S., Chee, C. Y., and Ho, R. C. (2020). Mental Health Strategies to Combat the Psychological Impact of COVID-19 Beyond Paranoia and Panic. Ann. Acad. Med. Singapore 49, 1-3.

Holmes, E. A., O’connor, R. C., Perry, H., Tracey, I., Wessely, S., Arseneault, L., et al. (2020). Position Paper Multidisciplinary research priorities for the COVID-19 pandemic: a call for action for mental health science. Lancet Psychiatry. doi:10.1016/S2215-0366(20)30168-1.

Husky, M. M., Kovess-Masfety, V., and Swendsen, J. D. (2020). Stress and anxiety among university students in France during Covid-19 mandatory confinement. Compr. Psychiatry 102, 152191. doi:10.1016/j.comppsych.2020.152191.

Jordanian Ministry of Health (2020). COVID-19 in Jordan. [Online]. Available at: https://corona.moh.gov.jo/ar [Accessed April 16, 2020].

Kessler, R. C., Andrews, G., Colpe, L. J., Hiripi, E., Mroczek, D. K., Normand, S. L. T., et al. (2002). Short screening scales to monitor population prevalences and trends in non-specific psychological distress. Psychol. Med. 32, 959-976. doi:10.1017/S0033291702006074.

Leung, J., Gartner, C., Hall, W., Lucke, J., and Dobson, A. (2012). A longitudinal study of the bidirectional relationship between tobacco smoking and psychological distress in a community sample of young Australian women. Psychol. Med. 42, 1273-1282. doi:DOI: $10.1017 /$ S0033291711002261.

National Comorbidity Survey (2013). National Comorbidity Survey: Arabic K10. [Online]. Available at: https://www.hcp.med.harvard.edu/ncs/k6_scales.php [Accessed May 2, 2020].

Olaimat, A. N., Aolymat, I., Elsahoryi, N., Shahbaz, H. M., and Holley, R. A. (2020). Attitudes, Anxiety, and Behavioral Practices Regarding COVID-19 among University Students in Jordan: A Cross-Sectional Study. Am. J. Trop. Med. Hyg. 103, 1177-1183. doi:10.4269/ajtmh.20-0418. 
515

516

517

518

519

520

521

522

523

524

525

526

527

528

529

530

531

532

533

\section{4}

535

536

537

538

539

540

541

542

543

544

545

546

547

Ornell, F., Schuch, J. B., Sordi, A. O., and Kessler, F. H. P. (2020). "Pandemic fear" and COVID-19: mental health burden and strategies. Brazilian J. Psychiatry 00, 000-000. doi:10.1590/15164446-2020-0008.

Pfefferbaum, B., and North, C. S. (2020). Mental Health and the Covid-19 Pandemic. N. Engl. J. Med. 383, 510-512. doi:10.1056/NEJMp2008017.

Prime Ministry of Jordan (2020). Official Reports. [Online]. Available at: http://www.pm.gov.jo/category/7603/خبار.html [Accessed April 17, 2020].

SAMHSA (2014). Coping With Stress During Infectious Disease Outbreaks. Available at: https://store.samhsa.gov/sites/default/files/d7/priv/sma14-4885.pdf [Accessed May 5, 2020].

Thornton, L., Batterham, P. J., Fassnacht, D. B., Kay-Lambkin, F., Calear, A. L., and Hunt, S. (2016). Recruiting for health, medical or psychosocial research using Facebook: Systematic review. Internet Interv. 4, 72-81. doi:10.1016/j.invent.2016.02.001.

USCF (2020). Emotional Well-Being and Coping During COVID-19. [Online]. Available at: https://psychiatry.ucsf.edu/copingresources/covid19.

World Health Organization (2020). Coronavirus disease (COVID-19) outbreak situation. [Online]. Available at: https://www.who.int/emergencies/diseases/novel-coronavirus-2019 [Accessed June 25, 2020].

\section{Data Availability Statement}

The dataset generated and analyzed for this study are available from the corresponding author on a reasonable request

\section{$2 \quad$ Figure Legends}

Figure 1. The levels of total K10 distress scores among different age groups of the respondents.

Figure 2. Distribution of students (by gender) within different distress categories based on the overall K10 score for each student.

Figure 3. Students' Motivation for Distance Learning per Distress Category

Figure 4. Reported medication use for coping with the COVID-19 related psychological distress among respondents (percentage)

Figure 5. Types of medications that were used by 49 students for coping with the COVID-19 induced psychological distress.

Figure 6. The issue of the greatest concern as perceived by the 381 students (percentage) 
549 Table 1. Sociodemographic characteristics of the Respondents $(\mathbf{n}=\mathbf{3 8 1})$

\begin{tabular}{l} 
Variables \\
\hline \multicolumn{1}{l}{ Sex } \\
Male \\
Female \\
Age (Mean, SD) \\
$18-22$ \\
$23-27$ \\
$28-32$ \\
$33-38$
\end{tabular}

Marital Status

Single

Married

Region of residence

Northern governorates

Central governorates

Southern governorates

Smoking Status

Current Smoker

Currently non-smoker

Academic Institution

Public university/college

Private university/college

Study Level

Undergraduate

Postgraduate

History of pre-existing psychiatric conditions

$$
\text { Yes }
$$

No

\section{Results}

$$
\begin{aligned}
& n=182(47.8 \%) \\
& n=199(52.2 \%) \\
& 22.6,3.16 \\
& n=208(54.6 \%) \\
& n=142(37.3 \%) \\
& n=22(5.8 \%) \\
& n=9(2.3 \%) \\
& n=352(92.4 \%) \\
& n=29(7.6 \%)
\end{aligned}
$$

$\mathrm{n}=60(15.7 \%)$

$\mathrm{n}=302(79.3 \%)$

$\mathrm{n}=19(5.0 \%)$

$\mathrm{n}=117(29.9 \%)$

$\mathrm{n}=267(\mathbf{7 0 . 1 \%})$

$$
\mathbf{n}=\mathbf{2 0 9}(\mathbf{5 4 . 9 \%})
$$$$
\mathrm{n}=172(\mathbf{4 5 . 1 \%})
$$

$\mathrm{n}=323(\mathbf{8 4 . 8 \%})$

$\mathrm{n}=\mathbf{5 8}(\mathbf{1 5 . 2 \%})$

$\mathrm{n}=15(3.9 \%)$

$\mathrm{n}=366(96.1 \%)$

Current use of medications among the 15 students who reported a history of pre-existing psychiatric conditions
Yes
$\mathrm{n}=\mathbf{8}$
No
$\mathrm{n}=7$ 
556 Table 2. The severity of Psychological distress among respondents based on K10 distress scale's 557 categorization.

\begin{tabular}{|l|c|c|c|}
\hline $\begin{array}{c}\text { K10 Psychological Distress } \\
\text { Category }\end{array}$ & $\begin{array}{c}\text { Total K10 } \\
\text { Score range }\end{array}$ & Frequency (n) & Percentage (\%) \\
\hline No Distress & $\mathbf{1 0 - 1 9}$ & 27 & 7.1 \\
\hline Mild Distress & $\mathbf{2 0 - 2 4}$ & 41 & 10.8 \\
\hline Moderate Distress & $\mathbf{2 5 - 2 9}$ & 48 & 12.6 \\
\hline Severe Distress & $\mathbf{3 0 - 5 0}$ & 265 & 69.5 \\
\hline \multicolumn{2}{|c|}{ Total } & $\mathbf{3 8 1}$ & $\mathbf{1 0 0}$ \\
\hline
\end{tabular}


Table 3. Results of Ordinal Logistic Regression for the correlation between psychological

579 distress severity and independent predictors.

Predictors

Crude OR [95\% CI]

$0.67[0.50-0.90]$

0.008

$0.64[0.44-0.94]$

0.022

Gender

Female

Reference

Male

$0.67[0.40-1.1]$

0.141

$0.56[0.30-1.03]$

0.063

\section{Smoking}

\begin{tabular}{lllll} 
No & Reference & \multicolumn{2}{l}{ Reference } \\
\hline Yes & $1.48[0.81-2.72]$ & 0.206 & $1.99[1.10-3.39]$ & $\mathbf{0 . 0 4 9}$
\end{tabular}

Study Level

\begin{tabular}{lllll} 
Postgraduate & Reference & \multicolumn{3}{l}{ Reference } \\
Undergraduate & $0.59[0.26-1.36]$ & 0.216 & $0.53[0.17-1.64]$ & 0.272
\end{tabular}

\section{University/College}

\begin{tabular}{lllll} 
Private & Reference & \multicolumn{3}{l}{ Reference } \\
\hline Public & $1.96[1.15-3.34]$ & 0.013 & $1.43[0.74-2.77]$ & 0.287
\end{tabular}

Motivation for

Distance learning

\begin{tabular}{ccccc}
\hline Low & Reference & \multicolumn{2}{l}{ Reference } \\
\hline No & $2.62[1.40-4.93]$ & 0.003 & $2.49[1.29-4.80]$ & $\mathbf{0 . 0 0 7}$ \\
\hline Moderate & $0.99[0.49-2.02]$ & 0.983 & $1.27[0.59-2.73]$ & 0.535 \\
\hline High/Strong & $0.08[0.01-0.76]$ & 0.028 & $0.10[0.01-0.98]$ & $\mathbf{0 . 0 4 8}$
\end{tabular}


Table 4. The degree of motivation for online distance learning among respondents.

\begin{tabular}{|c|c|c|}
\hline Degree of Motivation & Frequency (N) & Percentage (\%) \\
\hline No Motivation & 209 & 54.9 \\
\hline Low Motivation & 98 & 25.7 \\
\hline Moderate Motivation & 69 & 18.1 \\
\hline Strong Motivation & 5 & 1.3 \\
\hline Total & $\mathbf{3 8 1}$ & $\mathbf{1 0 0}$ \\
\hline
\end{tabular}

Table 5. Coping activities during the COVID-19 pandemic and the nationwide curfew in Jordan among the respondents.

\begin{tabular}{|c|c|c|}
\hline Coping Activity & Frequency $(\mathbf{n})$ & Percentage $(\%)$ \\
\hline $\begin{array}{l}\text { Spending more time on social networking platforms like } \\
\text { Facebook and Instagram }\end{array}$ & 269 & 70.6 \\
\hline Talking to friends on mobile phones and internet & 217 & 57 \\
\hline Watching television and movies & 210 & 55.1 \\
\hline More engagement with the family & 202 & 53 \\
\hline Listening to music & 162 & 42.5 \\
\hline Practicing sports at home & 113 & 29.7 \\
\hline Studying and preparing for exams & 102 & 26.8 \\
\hline Increase smoking & 69 & 18.1 \\
\hline Reading Books / Novels & 68 & 17.8 \\
\hline Meditation & 58 & 15.2 \\
\hline Herbal drinks & 57 & 15 \\
\hline Practicing Yoga & 6 & 1.6 \\
\hline Talking to a psychological counselor & 6 & 1.6 \\
\hline Others & 33 & 8.7 \\
\hline
\end{tabular}

590 Table 6. Medicinal drugs' usage frequency among the 49 students who reported the use of 591 different medications in response to the COVID-19 induced distress.

\begin{tabular}{|l|c|c|}
\hline \multicolumn{1}{|c|}{ Frequency of usage } & Number of students & Percentage (\%) \\
\hline 1-2 times in a month & 17 & 34.7 \\
\hline 1-2 times in a week & 13 & 26.5 \\
\hline 3-4 times in a week & 10 & 20.4 \\
\hline Everyday Total & 9 & 18.4 \\
\hline \multicolumn{1}{|c|}{ To } & $\mathbf{1 0 0}$ \\
\hline
\end{tabular}


Figures

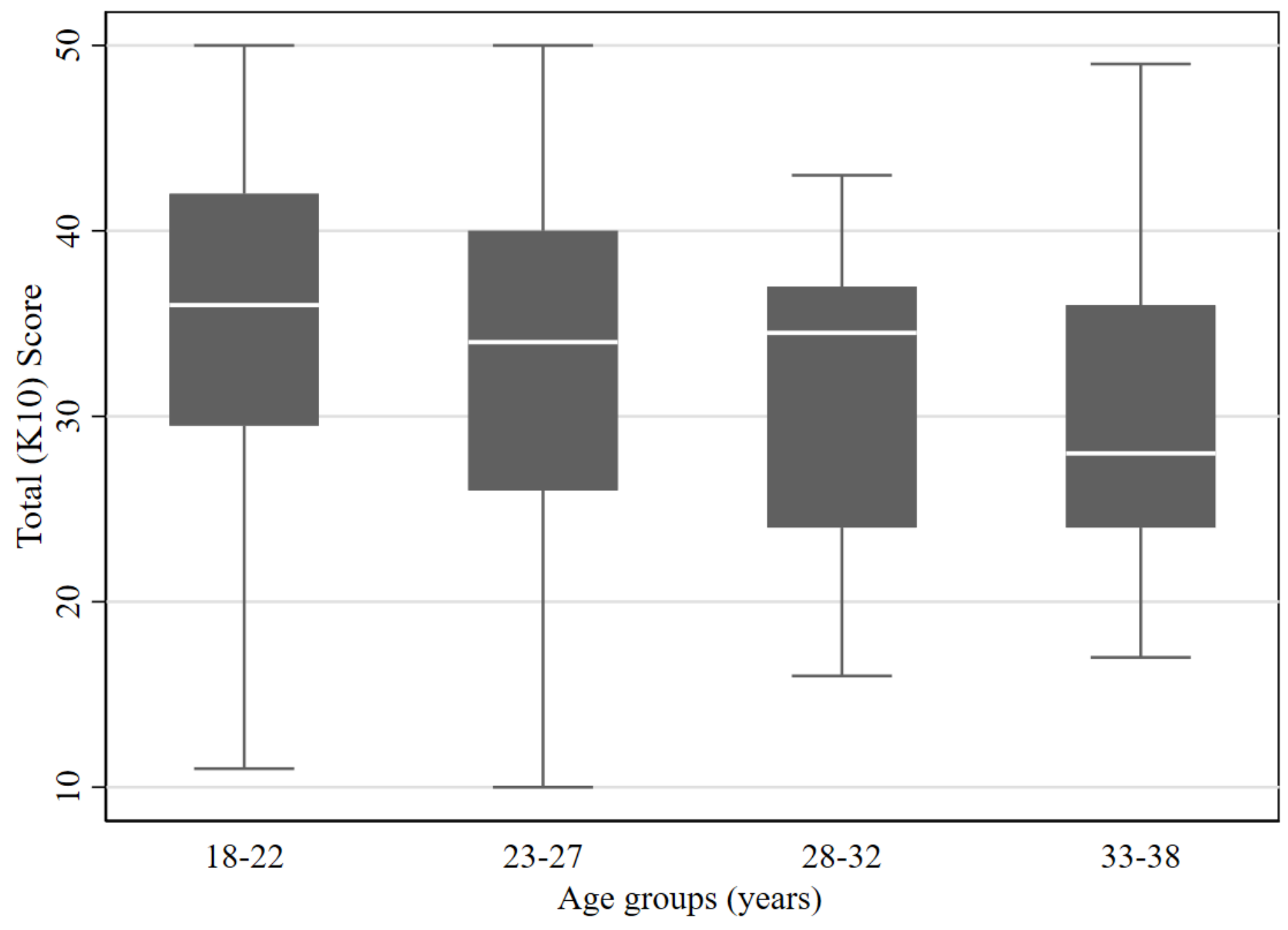

Figure 1

The levels of total K10 distress scores among different age groups of the respondents. 


\section{Distribution of Students (by gender) within Different Distress Categories}

\section{0}

250

200

150

100

50

0

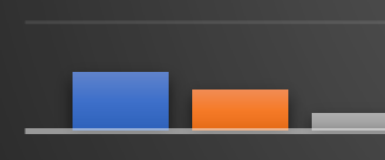

No Distress

Mild Distress

Moderate Distress

Severe Distress

Total

- Male

口 Female

Figure 2

Distribution of students (by gender) within different distress categories based on the overall K10 score for each student. 


\section{Students' Motivation for Distance Learning by Distress Category}

200

150

100

50

0

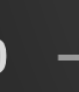

No Distress

Low Distress Moderate Distress

Severe Distress

$\square$ No Motivation $\square$ Low Motivation $\square$ Moderate Motivation $\square$ Strong Motivation

\section{Figure 3}

Students' Motivation for Distance Learning per Distress Category 


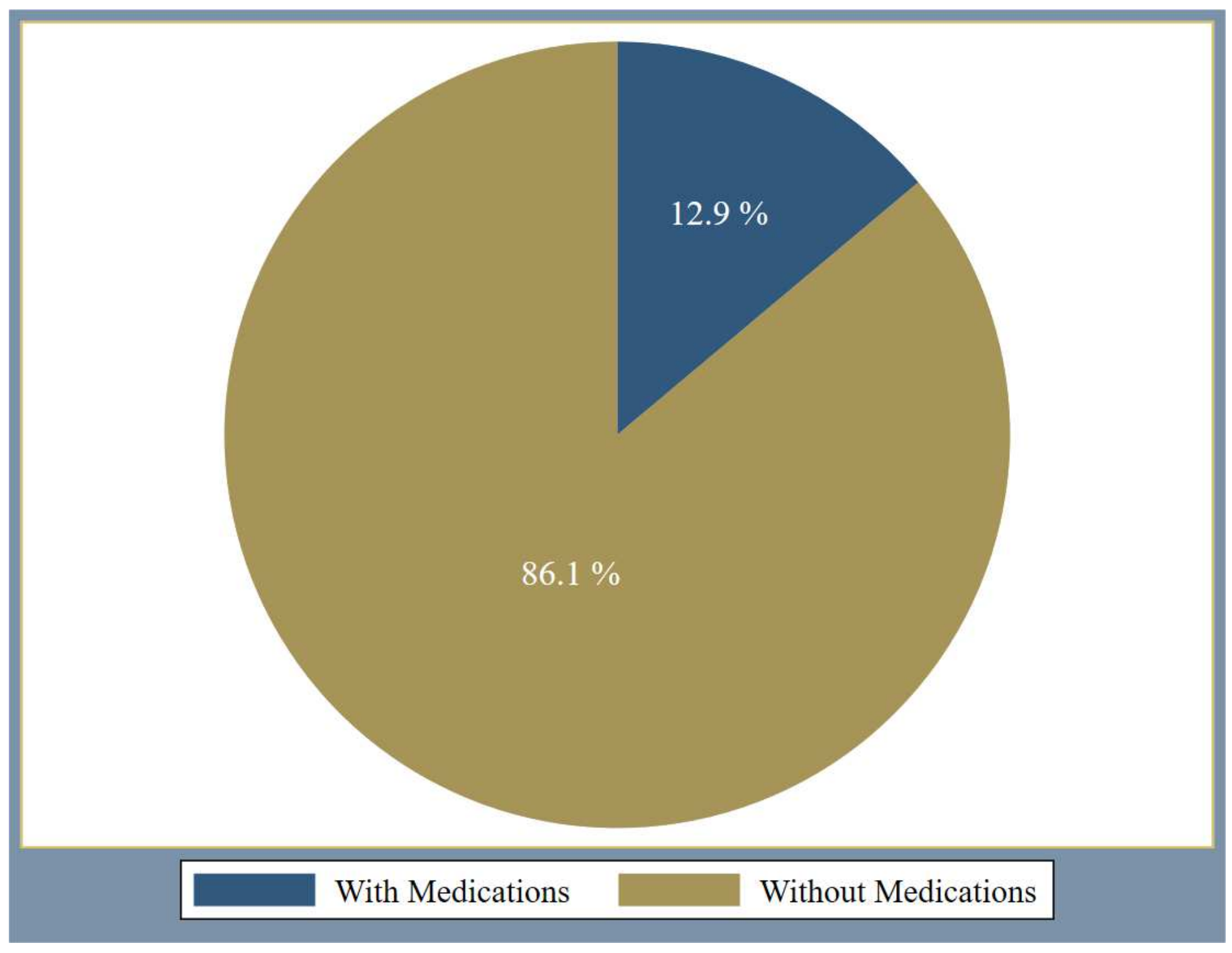

\section{Figure 4}

Reported medication use for coping with the COVID-19 related psychological distress among respondents (percentage) 


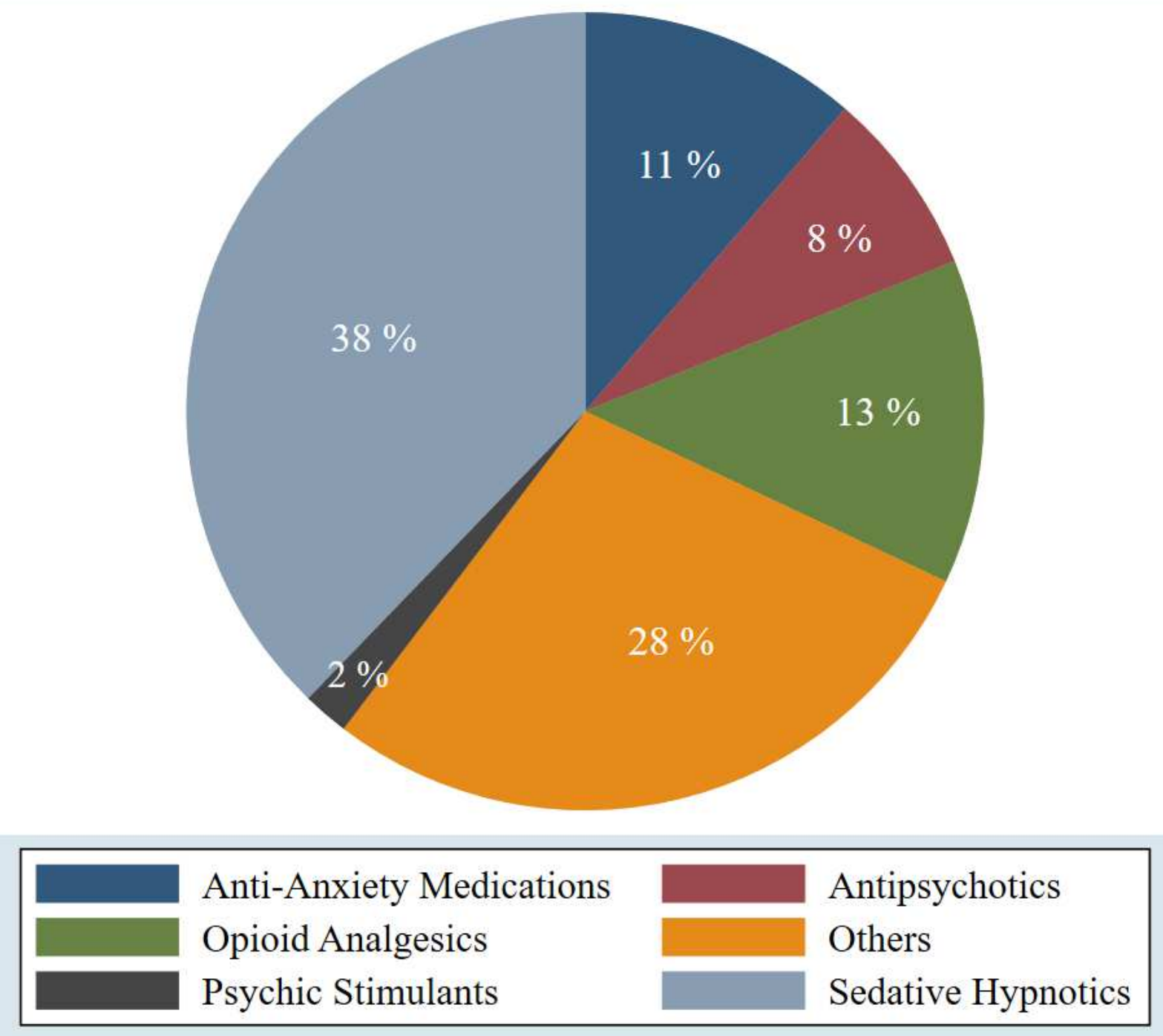

\section{Figure 5}

Types of medications that were used by 49 students for coping with the COVID-19 induced psychological distress. 


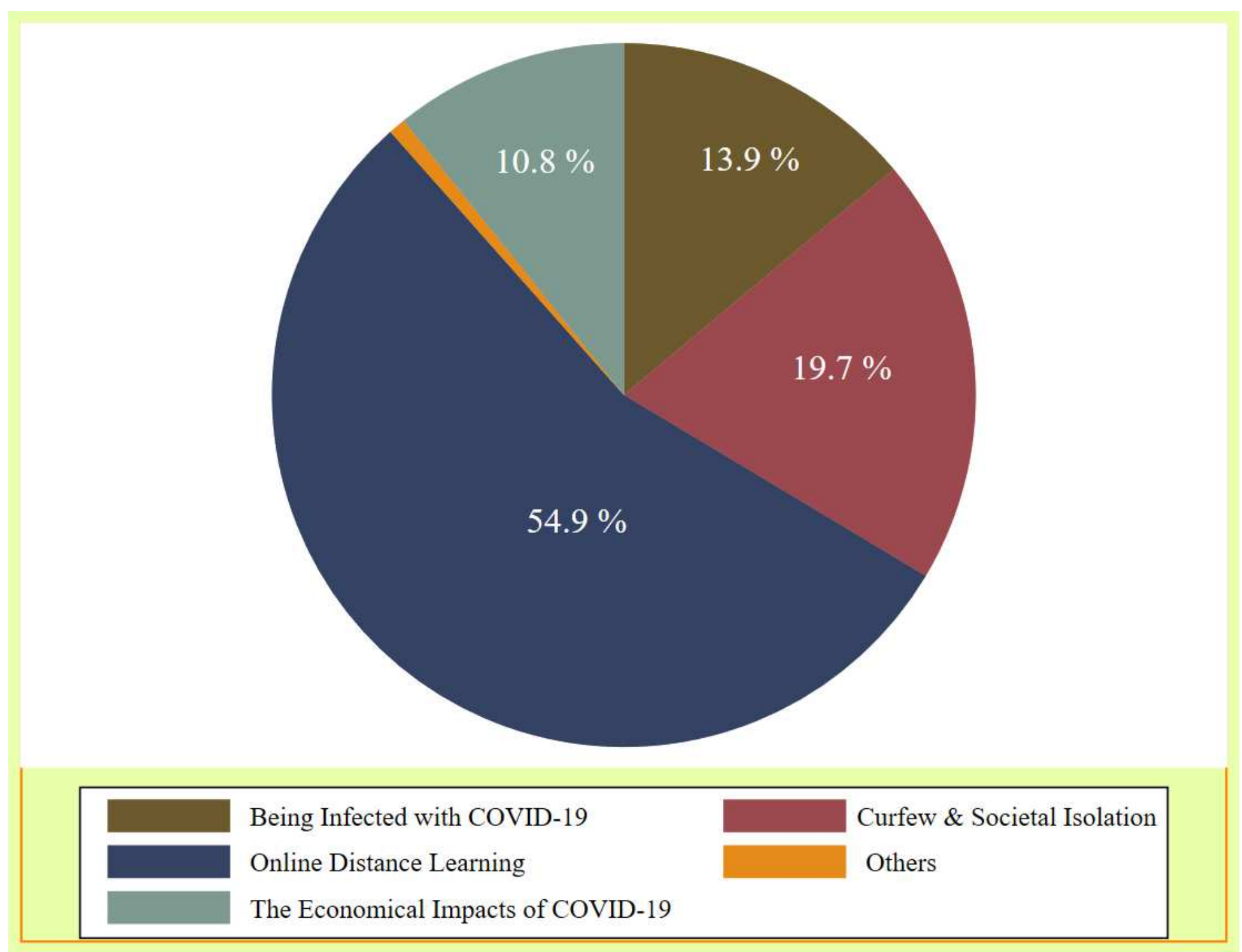

\section{Figure 6}

The issue of the greatest concern as perceived by the 381 students (percentage) 